\title{
Weakly Laskerian modules and weak cofiniteness
}

\author{
Bahram Vakili and Jafar Azami
}




\title{
WEAKLY LASKERIAN MODULES AND WEAK COFINITENESS
}

\author{
BAHRAM VAKILI AND JAFAR AZAMI
}

Received 18 September, 2012

\begin{abstract}
Let $R$ be a commutative Noetherian ring, $\mathfrak{a}$ an ideal of $R$. It is shown that if $\mathfrak{a}=$ $\left(x_{1}, \ldots, x_{t}\right)$, and $M$ is an $R$-module, then $\operatorname{Ext}_{R}^{i}(R / \mathfrak{a}, M)$ is weakly Laskerian for all $i$ iff $\operatorname{Tor}_{i}^{R}(R / \mathfrak{a}, M)$ is weakly Laskerian for all $i$ iff the Koszul cohomology module $H^{i}\left(x_{1}, \ldots, x_{t} ; M\right)$ is weakly Laskerian for all $i$. Furthermore, each of these coditions imply that $M / \mathfrak{a}^{n} M$ is weakly Laskerian for all $n \in \mathbb{N}$. In Section 3, we show that if $M$ is an $R$-module with Supp $M \subseteq V(\mathfrak{a})$, then $M$ is a-weakly cofinite, in the following cases:

a) there exists $x \in \mathfrak{a}$ such that $0: M x$ and $M / x M$ are both a-weakly cofinite.

b) there exists $x \in \sqrt{\mathfrak{a}}$ such that $0: M x$ and $M / x M$ are both weakly Laskerian.
\end{abstract}

2010 Mathematics Subject Classification: 13D45; 13E05

Keywords: weakly Laskerian modules, a-weakly cofinite modules, local cohomology

\section{INTRODUCTION}

Throughout this paper, $R$ will always be a commutative Noetherian ring with nonzero identity, and $\mathfrak{a}$ will be an ideal of $R$. Let $M$ be an $R$-module. The $\mathfrak{a}$-torsion submodule of $M$ is defined as $\Gamma_{\mathfrak{a}}(M)=\bigcup_{n \geq 1}\left(0:_{M} \mathfrak{a}^{n}\right)$. The $i^{\text {th }}$ local cohomology functor $\mathrm{H}_{\mathfrak{a}}^{i}($.$) is defined as the i^{\text {th }}$ right derived functor $\Gamma_{\mathfrak{a}}($.$) . It is known that for$ each $i \geq 0$ there is a natural isomorphism of $R$-modules

$$
\mathrm{H}_{\mathfrak{a}}^{i}(M) \cong \underset{n \geq 1}{\lim } \operatorname{Ext}_{R}^{i}\left(R / \mathfrak{a}^{n}, M\right)
$$

We refer the reader to [5] or [1] for the basic properties of local cohomology.

The notions of weakly Laskerian modules and a-weakly cofinite modules were introduced by Divaani-Aazar and Mafi in [3] and [4]. An $R$ module $M$ is said to be weakly Laskerian if the set of associated primes of any quotient module of $M$ is finite. An $R$ module $M$ is said to be $\mathfrak{a}$-weakly cofinite if Supp $M \subseteq V(\mathfrak{a})$ and $\operatorname{Ext}_{R}^{i}(R / \mathfrak{a}, M)$ is weakly Laskerian for all $i \geq 0$.

Divaani-Aazar and Mafi in [4, Theorem 2.10 ] have shown using change of rings principle and spectral sequence that if $M$ is an a-weakly cofinite $R$-module, then

The research of the first author was supported in part by a grant from Islamic Azad University, Shabestar Branch. 
$M / \mathfrak{a} M$ is weakly Laskerian. In Section 2 , without using change of rings principle and spectral sequence, we prove that if $M$ is an $R$-module such that $\operatorname{Ext}_{R}^{i}(R / \mathfrak{a}, M)$ is a weakly Laskerian $R$-module for all $i \geq 0$, then $M / \mathfrak{a}^{n} M$ is weakly Laskerian for all $n \in \mathbb{N}$. One of the main results of this article is to prove that if $\mathfrak{a}=\left(x_{1}, \ldots, x_{t}\right)$, and $M$ is an $R$-module, then the following statements are equivalent:

(ii) $\operatorname{Ext}_{R}^{i}(R / \mathfrak{a}, M)$ is a weakly Laskerian $R$-module for all $i$.

(ii) $\operatorname{Tor}_{i}^{R}(R / \mathfrak{a}, M)$ is a weakly Laskerian $R$-module for all $i$.

(iii) The Koszul cohomology module $H^{i}\left(x_{1}, \ldots, x_{t} ; M\right)$ is weakly Laskerian $R$ module for all $i$.

In Section 3, we obtain a sufficient condition for a-weakly cofinite modules. In fact, we prove that if $M$ is an $R$-module with $\operatorname{Supp} M \subseteq V(\mathfrak{a})$, then $M$ is a-weakly cofinite, in the following cases:

a) there exists $x \in \mathfrak{a}$ such that $0: M x$ and $M / x M$ are both a-weakly cofinite.

b) there exists $x \in \sqrt{\mathfrak{a}}$ such that $0: M x$ and $M / x M$ are both weakly Laskerian.

In Section 4, we prove that if $\mathfrak{b}$ is a second ideal of $R$ with $\mathfrak{b} \supseteq \mathfrak{a}$ and $\operatorname{cd}(\mathfrak{b})=1$ and $M$ is a weakly Laskerian $R$-module, then for every finitely generated $R$-module $L$ with Supp $L \subseteq V(\mathfrak{b})$, the $R$-module $\operatorname{Ext}_{\mathrm{R}}^{\mathrm{j}}\left(\mathrm{L}, \mathrm{H}_{\mathfrak{a}}^{\mathrm{i}}(\mathrm{M})\right)$ is weakly Laskerian for all $i$ and $j$. In particular, the $R$-module $H_{\mathfrak{a}}^{i}(M) / \mathfrak{b}^{n} H_{\mathfrak{a}}^{i}(M)$ is weakly Laskerian for all $i$ and $n$.

\section{WEAKLY LASKERIAN MODULES AND $\mathfrak{a}$-WEAKLY COFINITE MODULES}

To prove the main results of this paper, we need to the following two lemmas.

Lemma 1. Let $M$ be an $R$-module such that $0: M$ a is a weakly Laskerian $R$ module. Then $0: M \mathfrak{a}^{n}$ is weakly Laskerian for all $n \in \mathbb{N}$.

Proof. Consider the exact sequence

$$
0 \rightarrow 0: M \mathfrak{a} \rightarrow 0:_{M} \mathfrak{a}^{n} \stackrel{f}{\rightarrow} a_{1}\left(0::_{M} \mathfrak{a}^{n}\right) \oplus \cdots \oplus a_{t}\left(0:_{M} \mathfrak{a}^{n}\right),
$$

where $\mathfrak{a}=\left(a_{1}, \ldots, a_{t}\right)$ and $f$ is defined by $f(x)=\left(a_{1} x, \ldots, a_{t} x\right)$. The result is followed by induction on $n$ and [3, Lemma 2.3 (i)]. Note that $a_{i}\left(0: M \mathfrak{a}^{n}\right)$ is a submodule of $0: M \mathfrak{a}^{n-1}$ for all $i=1,2, \ldots, t$.

Lemma 2. Let $M$ be an $R$-module such that $M / \mathfrak{a} M$ is a weakly Laskerian $R$ module. Then $M / \mathfrak{a}^{n} M$ is weakly Laskerian for all $n \in \mathbb{N}$.

Proof. Consider the exact sequence

$$
\left(M / \mathfrak{a}^{n-1} M\right)^{t} \stackrel{f}{\rightarrow} M / \mathfrak{a}^{n} M \stackrel{g}{\rightarrow} M / \mathfrak{a} M \rightarrow 0,
$$

where $\mathfrak{a}=\left(a_{1}, \ldots, a_{t}\right), g$ is the canonical map, and $f$ is defined by

$$
f\left(m_{1}+\mathfrak{a}^{n-1} M, \cdots, m_{t}+\mathfrak{a}^{n-1} M\right)=a_{1} m_{1}+\cdots+a_{t} m_{t}+\mathfrak{a}^{n} M .
$$


Now, the result is followed by induction on $n$ and [3, Lemma 2.3 (i)].

Divaani-Aazar and Mafi in [4, Theorem 2.10] have shown using change of rings principle and spectral sequence that if $M$ is an a-weakly cofinite $R$-module, then $M / \mathfrak{a} M$ is weakly Laskerian. We generalize this result and give a direct proof without using change of rings principle and spectral sequence.

Theorem 1. Let $M$ be an $R$-module such that $\operatorname{Ext}_{R}^{i}(R / \mathfrak{a}, M)$ is a weakly Laskerian $R$-module for all $i \geq 0$. Then $M / \mathfrak{a}^{n} M$ is weakly Laskerian for all $n \in \mathbb{N}$.

Proof. By Lemma 2, it is enough to prove that $M / \mathfrak{a} M$ is weakly Laskerian. To do this, let $\mathfrak{a}=\left(x_{1}, \ldots, x_{n}\right)$. Then $M / \mathfrak{a} M \simeq H^{n}\left(x_{1}, \ldots, x_{n} ; M\right)$, where

$H^{n}\left(x_{1}, \ldots, x_{n} ; M\right)$ denotes the $n^{\text {th }}$ Koszul cohomology module. Consider the coKoszul complex

$$
\begin{aligned}
K^{\bullet}(\mathbf{x}, M): 0 \rightarrow \operatorname{Hom}\left(K_{0}(\mathbf{x}), M\right) \rightarrow \operatorname{Hom}\left(K_{1}(\mathbf{x}), M\right) & \rightarrow \cdots \\
& \rightarrow \operatorname{Hom}\left(K_{n}(\mathbf{x}), M\right) \rightarrow 0 .
\end{aligned}
$$

Then $H^{i}\left(x_{1}, \cdots, x_{n} ; M\right)=Z^{i} / B^{i}$, where $B^{i}$ and $Z^{i}$ are the modules of coboundaries and cocycles of the complex $K^{\bullet}(\mathbf{x}, M)$, respectively. Let $\mathcal{W}$ be the class of all $R$ modules $N$ such that $\operatorname{Ext}_{R}^{i}(R / \mathfrak{a}, N)$ is weakly Laskerian for all $i \geq 0$. By induction we claim that $B^{j} \in \mathcal{W}$ for all $j$. We have $B^{0}=0 \in \mathcal{W}$. Now, let $B^{t} \in$ W. Put $C^{i}=\operatorname{Hom}\left(K_{i}(\mathbf{x}), M\right) / B^{i}$. Since $K_{t}(\mathbf{x})$ is a finitely generated free $R$ module, it follows that $\operatorname{Hom}\left(K_{t}(\mathbf{x}), M\right)$ is a direct sum of finitely many copies of $M$. Therefore, $\operatorname{Hom}\left(K_{t}(\mathbf{x}), M\right) \in \mathcal{W}$ by [3, Lemma 2.3 (i)]. Now, since $B^{t} \in \mathcal{W}$ and $\operatorname{Hom}\left(K_{t}(\mathbf{x}), M\right) \in \mathcal{W}$, we have $C^{t} \in \mathcal{W}$ by [3, Lemma 2.3 (i)]. Hence $0: C^{t} \mathfrak{a} \simeq$ $\operatorname{Hom}_{R}\left(R / \mathfrak{a}, C^{t}\right)$ is weakly Laskerian. But since $\mathfrak{a} H^{t}\left(x_{1}, \ldots, x_{n} ; M\right)=0$, it follows that $H^{t}\left(x_{1}, \ldots, x_{n} ; M\right) \subseteq 0:_{C^{t}} \mathfrak{a}$, and so $H^{t}\left(x_{1}, \ldots, x_{n} ; M\right)$ is weakly Laskerian. Next, from the short exact sequence

$$
0 \rightarrow H^{t}\left(x_{1}, \ldots, x_{n} ; M\right) \rightarrow C^{t} \rightarrow B^{t+1} \rightarrow 0
$$

and [3, Lemma 2.3 (i)] we deduce that $B^{t+1} \in \mathcal{W}$. Hence, by induction we have proved that $B^{j} \in \mathcal{W}$ for all $j$. Now, since $B^{n} \in \mathcal{W}$ and $\operatorname{Hom}\left(K_{n}(\mathbf{x}), M\right) \in \mathcal{W}$, we obtain that $C^{n} \in \mathcal{W}$. Hence $0: C^{n} \mathfrak{a} \simeq \operatorname{Hom}_{R}\left(R / \mathfrak{a}, C^{n}\right)$ is weakly Laskerian. Thus $H^{n}\left(x_{1}, \ldots, x_{n} ; M\right) \subseteq 0:_{C^{n}} \mathfrak{a}$ implies that $H^{n}\left(x_{1}, \ldots, x_{n} ; M\right)$ is weakly Laskerian. Since $M / \mathfrak{a} M=H^{n}\left(x_{1}, \ldots, x_{n} ; M\right)$, it follows that $M / \mathfrak{a} M$ is weakly Laskerian.

Corollary 1. Let $M$ be a a-weakly cofinite R-module. Then $M / \mathfrak{a}^{n} M$ is weakly Laskerian for all $n \in \mathbb{N}$.

Proof. The assertion follows from the definition and Theorem 1. 
Corollary 2. Let $\mathfrak{a}$ be an ideal of $R$, and let $M$ be an $R$-module such that $\mathrm{H}_{\mathfrak{a}}^{i}(M)$ is $\mathfrak{a}$-weakly cofinite for all $i$. Then $\operatorname{Ext}_{R}^{i}(R / \mathfrak{a}, M)$ is weakly Laskerian for all $i$, and $M / \mathfrak{a}^{n} M$ is weakly Laskerian for all $n \in \mathbb{N}$.

Proof. By Theorem 1, it is sufficient to prove that $\operatorname{Ext}_{R}^{i}(R / \mathfrak{a}, M)$ is weakly Laskerian for all $i$. The case $i=0$ is clear, so let $i>0$ and we do induction on $i$. We first reduce to the case $\Gamma_{\mathfrak{a}}(M)=0$. To do this, let $\bar{M}=M / \Gamma_{\mathfrak{a}}(M)$, then we have the exact sequence

$$
\begin{aligned}
\ldots \rightarrow \operatorname{Ext}_{R}^{i}\left(R / \mathfrak{a}, \Gamma_{\mathfrak{a}}(M)\right) \rightarrow \operatorname{Ext}_{R}^{i}(R / \mathfrak{a}, M) \rightarrow \operatorname{Ext}_{R}^{i}(R / \mathfrak{a}, \bar{M}) \\
\rightarrow \operatorname{Ext}_{\mathrm{R}}^{\mathrm{i}+1}\left(R / \mathfrak{a}, \Gamma_{\mathfrak{a}}(M)\right) \rightarrow \ldots
\end{aligned}
$$

and isomorphism $\mathrm{H}_{\mathfrak{a}}^{i}(M) \cong \mathrm{H}_{\mathfrak{a}}^{i}(\bar{M})$ for all $i>0$. Since $\Gamma_{\mathfrak{a}}(M)$ is $\mathfrak{a}$-weakly cofinite, so in view of [3, Lemma 2.3 (i)], we may assume that $M$ is $\mathfrak{a}$-torsion free. Let $E$ be the injective envelope of $M$ and put $L=E / M$. Then $H_{\mathfrak{a}}^{i}(E)=0$, and we therefore get the isomorphisms $\mathrm{H}_{\mathfrak{a}}^{i}(L) \cong \mathrm{H}_{\mathfrak{a}}^{i+1}(M)$ and $\operatorname{Ext}_{R}^{i}(R / \mathfrak{a}, L) \cong \operatorname{Ext}_{R}^{i+1}(R / \mathfrak{a}, M)$ for all $i \geq 0$. Now the assertion follows by induction.

Theorem 2. Let $\mathfrak{a}=\left(x_{1}, \ldots, x_{t}\right)$ be an ideal of $R$, and let $M$ be an $R$-module. Then the following statements are equivalent:

(i) $\operatorname{Ext}_{R}^{i}(R / \mathfrak{a}, M)$ is a weakly Laskerian $R$-module for all $i$.

(ii) $\operatorname{Tor}_{i}^{R}(R / \mathfrak{a}, M)$ is a weakly Laskerian $R$-module for all $i$.

(iii) The Koszul cohomology module $H^{i}\left(x_{1}, \ldots, x_{t} ; M\right)$ is weakly Laskerian $R$ module for all $i$.

Furthermore, each of these coditions imply that $M / \mathfrak{a}^{n} M$ is weakly Laskerian for all $n \in \mathbb{N}$.

Proof. (i) $\Rightarrow$ (ii) Let

$$
\mathbb{F}_{\bullet}: \cdots \rightarrow F_{2} \rightarrow F_{1} \rightarrow F_{0} \rightarrow 0
$$

be a free resolution of finitely generated $R$-modules for $R / \mathfrak{a}$. We have $\operatorname{Tor}_{i}^{R}(R / \mathfrak{a}, M)=Z_{i} / B_{i}$, where $B_{i}$ and $Z_{i}$ are the modules of boundaries and cycles of the complex $\mathbb{F}_{\bullet} \otimes_{R} M$, respectively. Let $W$ be the class of all $R$ modules $N$ such that $\operatorname{Ext}_{R}^{i}(R / \mathfrak{a}, N)$ is weakly Laskerian for all $i \geq 0$. By induction we claim that $Z_{j} \in \mathcal{W}$ for all $j$. We have $Z_{0}=F_{0} \otimes_{R} M \in \mathcal{W}$. Now, let $Z_{t} \in \mathcal{W}$. Consider the exact sequence

$$
(\dagger) \quad 0 \rightarrow C_{i+1} \rightarrow Z_{i} \rightarrow \operatorname{Tor}_{i}^{R}(R / \mathfrak{a}, M) \rightarrow 0,
$$

where $C_{i}=F_{i} \otimes_{R} M / Z_{i}$. Hence we obtain the exact sequence

$$
Z_{i} / \mathfrak{a} Z_{i} \rightarrow \operatorname{Tor}_{i}^{R}(R / \mathfrak{a}, M) \rightarrow 0 .
$$

Therefore, $\operatorname{Tor}_{t}^{R}(R / \mathfrak{a}, M)$ is a homomorphic image of $Z_{t} / \mathfrak{a} Z_{t}$. Since $Z_{t} \in \mathcal{W}$, it follows from Theorem 1 that $Z_{t} / \mathfrak{a} Z_{t}$ is weakly Laskerian, and so $\operatorname{Tor}_{t}^{R}(R / \mathfrak{a}, M)$ 
is weakly Laskerian. Hence, we deduce by $(\dagger)$ that $C_{t+1} \in \mathcal{W}$, and so $Z_{t+1} \in \mathcal{W}$. Hence by induction we have proved that $Z_{j} \in \mathcal{W}$ for all $j$. It follows from Theorem 1

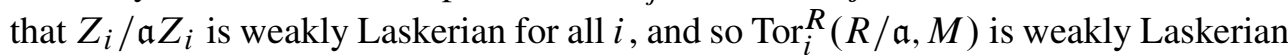
for all $i$.

To prove the implication (ii) $\Rightarrow$ (iii), since

$$
H^{i}\left(x_{1}, \ldots, x_{t} ; M\right) \simeq H_{t-i}\left(x_{1}, \ldots, x_{t} ; M\right),
$$

so it is sufficient to show that $H_{i}\left(x_{1}, \ldots, x_{t} ; M\right)$ is weakly Laskerian for all $i$. Let $\mathbf{x}=x_{1}, \ldots, x_{t}$. Consider the Koszul complex

$$
K_{\bullet}(\mathbf{x}): 0 \rightarrow K_{t}(\mathbf{x}) \rightarrow K_{t-1}(\mathbf{x}) \rightarrow \cdots \rightarrow K_{1}(\mathbf{x}) \rightarrow K_{0}(\mathbf{x}) \rightarrow 0,
$$

We have $H_{i}\left(x_{1}, \ldots, x_{t} ; M\right)=Z_{i} / B_{i}$, where $B_{i}$ and $Z_{i}$ are the modules of boundaries and cycles of the complex $K_{\bullet}(\mathbf{x}) \otimes_{R} M$, respectively. Let $\mathcal{W}$ be the class of all $R$ modules $N$ such that $\operatorname{Tor}_{i}^{R}(R / \mathfrak{a}, N)$ is weakly Laskerian for all $i \geq 0$. Consider the exact sequence

$$
0 \rightarrow C_{i+1} \rightarrow Z_{i} \rightarrow H_{i}\left(x_{1}, \ldots, x_{t} ; M\right) \rightarrow 0,
$$

where $C_{i}=K_{i}(\mathbf{x}) \otimes_{R} M / Z_{i}$. Hence we obtain the exact sequence

$$
Z_{i} / \mathfrak{a} Z_{i} \rightarrow H_{i}\left(x_{1}, \ldots, x_{t} ; M\right) \rightarrow 0 .
$$

By using a similar proof as in the proof of the implication (i) $\Rightarrow$ (ii), $Z_{i} \in \mathcal{W}$ for all $i$. It follows that $Z_{i} / \mathfrak{a} Z_{i}=\operatorname{Tor}_{0}^{R}\left(R / \mathfrak{a}, Z_{i}\right)$ is weakly Laskerian for all $i$, and so $H_{i}\left(x_{1}, \ldots, x_{t} ; M\right)$ is weakly Laskerian for all $i$.

To prove the implication (iii) $\Rightarrow$ (i), let

$$
\mathbb{F}_{\bullet}: \cdots \rightarrow F_{2} \rightarrow F_{1} \rightarrow F_{0} \rightarrow 0
$$

be a free resolution of finitely generated $R$-modules for $R / \mathfrak{a}$. We have $\operatorname{Ext}_{R}^{i}(R / \mathfrak{a}, M)$ $=Z^{i} / B^{i}$, where $B^{i}$ and $Z^{i}$ are the modules of coboundaries and cocycles of the complex $\operatorname{Hom}_{R}\left(\mathbb{F}_{\bullet}, M\right)$, respectively. Let $W$ be the class of all $R$ modules $N$ such that $H^{i}\left(x_{1}, \ldots, x_{t} ; N\right)$ is weakly Laskerian for all $i \geq 0$. Consider the short exact sequence

$$
0 \rightarrow \operatorname{Ext}_{R}^{i}(R / \mathfrak{a}, M) \rightarrow C^{i} \rightarrow B^{i+1} \rightarrow 0,
$$

where $C^{i}=\operatorname{Hom}_{R}\left(F_{i}, M\right) / B^{i}$. By using a similar proof as in the proof of the Theorem $1, B^{i} \in \mathcal{W}$ for all $i$. Thus $C^{i} \in \mathcal{W}$ for all $i$. Now, since

$$
\operatorname{Ext}_{R}^{i}(R / \mathfrak{a}, M) \subseteq 0:_{C^{i}} \mathfrak{a} \simeq \operatorname{Hom}_{R}\left(R / \mathfrak{a}, C^{i}\right) \simeq H^{0}\left(x_{1}, \ldots, x_{t} ; C^{i}\right)
$$

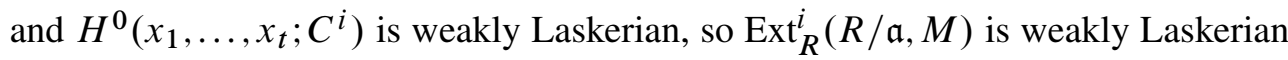
for all $i$.

Finally, the end part is followed by Theorem 1 . 
The first part of the next result has been proved using Gruson's Theorem by Divaani-Aazar and Mafi [4, Theorem 2.8 ] by using the same proof as that used in Delfino and Marley [2, Proposition 1]. We give a direct proof for this.

Theorem 3. Let $M$ be an $R$-module such that $\operatorname{Ext}_{R}^{i}(R / \mathfrak{a}, M)$ is a weakly Laskerian $R$-module for all $i \geq 0$. Then for any finitely generated $R$-module $L$ with Supp $L \subseteq V(\mathfrak{a})$, the $R$-modules $\operatorname{Ext}_{R}^{i}(L, M)$ and $\operatorname{Tor}_{i}^{R}(L, M)$ are weakly Laskerian for all $i \geq 0$.

Proof. We have $V\left(\operatorname{Ann}_{R} L\right)=\operatorname{Supp} L \subseteq V(\mathfrak{a})$. Hence there exists $n \in \mathbb{N}$ such that $\mathfrak{a}^{n} L=0$. It follows that $\mathfrak{a}^{n} \operatorname{Ext}_{R}^{i}(L, M)=0$ and $\mathfrak{a}^{n} \operatorname{Tor}_{i}^{R}(L, M)=0$ for all $i$. Let

$$
\mathbb{F}_{\bullet}: \cdots \rightarrow F_{2} \rightarrow F_{1} \rightarrow F_{0} \rightarrow 0
$$

be a free resolution of finitely generated $R$-modules for $\mathrm{L}$. Then $\operatorname{Ext}_{R}^{i}(L, M)$ $=Z^{i} / B^{i}$, where $B^{i}$ and $Z^{i}$ are the modules of coboundaries and cocycles of the complex $\operatorname{Hom}_{R}\left(\mathbb{F}_{\bullet}, M\right)$, respectively. Let $\mathcal{C}$ be the class of all $R$ modules $N$ such that $\operatorname{Ext}_{R}^{i}(R / \mathfrak{a}, N)$ is weakly Laskerian for all $i \geq 0$. Consider the short exact sequence

$$
0 \rightarrow \operatorname{Ext}_{R}^{i}(L, M) \rightarrow C^{i} \rightarrow B^{i+1} \rightarrow 0,
$$

where $C^{i}=\operatorname{Hom}_{R}\left(F_{i}, M\right) / B^{i}$. By using a similar proof as in the proof of the Theorem 1 and using Lemma 1, we have that $B^{i} \in \mathcal{C}$ for all $i$. (Note that $\operatorname{Ext}_{R}^{i}(L, M) \subseteq$ $0:_{C^{i}} \mathfrak{a}^{n}$.) Thus $C^{i} \in \mathcal{C}$ for all $i$. Hence $0:_{C^{i}} \mathfrak{a}$ is weakly Laskerian for all $i$, and so it follows from Lemma 1 that $0:_{C^{i}} \mathfrak{a}^{n}$ is weakly Laskerian for all $i$. Now, since $\operatorname{Ext}_{R}^{i}(L, M) \subseteq 0:_{C^{i}} \mathfrak{a}^{n}, \operatorname{Ext}_{R}^{i}(L, M)$ is weakly Laskerian for all $i$.

Also, we have $\operatorname{Tor}_{i}^{R}(L, M)=Z_{i} / B_{i}$, where $B_{i}$ and $Z_{i}$ are the modules of boundaries and cycles of the complex $\mathbb{F}_{\bullet} \otimes_{R} M$, respectively. Let $\ell^{\prime}$ be the class of all $R$ modules $N$ such that $\operatorname{Tor}_{i}^{R}(R / \mathfrak{a}, N)$ is weakly Laskerian for all $i \geq 0$. In view of Theorem 2 and our assumption, $M \in C^{\prime}$. Consider the exact sequence

$$
0 \rightarrow C_{i+1} \rightarrow Z_{i} \rightarrow \operatorname{Tor}_{i}^{R}(L, M) \rightarrow 0,
$$

where $C_{i}=F_{i} \otimes_{R} M / Z_{i}$. As $\mathfrak{a}^{n} \operatorname{Tor}_{i}^{R}(L, M)=0$ for all $i$, we obtain the exact sequence

$$
Z_{i} / \mathfrak{a}^{n} Z_{i} \rightarrow \operatorname{Tor}_{i}^{R}(L, M) \rightarrow 0 .
$$

Now, by using a similar proof as in the proof of the Theorem $2($ (i) $\Rightarrow$ (ii)) and using Lemma 2, we have $Z_{i} \in \mathcal{C}$ for all $i$. Therefore, it follows from Lemma 2 that $Z_{i} / \mathfrak{a}^{n} Z_{i}$ is weakly Laskerian for all $i$, and $\operatorname{Tor}_{i}^{R}(L, M)$ is weakly Laskerian for all $i$.

The change of ring principle for weak cofiniteness has been proved by using a spectral sequence argument by Divaani-Aazar and Mafi [4, Theorem 2.9 ]. We give a direct proof for it. 
Theorem 4. Let the ring $T$ be a homomorphic image of $R$, and let $M$ be a $T$ module. Then $M$ is an $\mathfrak{a} T$-weakly cofinite as a T-module if and only if $M$ is an $\mathfrak{a}$-weakly cofinite as an $R$-module.

Proof. Assume that $T=R / I$ for some ideal $I$ of $R$ and let $N$ be a $T$-module. Then $\mathfrak{p} \in \operatorname{Ass}_{R} N$ if and only if $\mathfrak{p} / I \in \operatorname{Ass}_{T} N$, and so $N$ is weakly Laskerian as a $T$-module if and only if $N$ is weakly Laskerian as an $R$-module. Also, since $\mathfrak{p} \in$ $\operatorname{Supp}_{R} N$ if and only if $\mathfrak{p} / I \in \operatorname{Supp}_{T} N$, it follows that $\operatorname{Supp}_{T} M \subseteq V(\mathfrak{a} T)$ if and only if $\operatorname{Supp}_{R} M \subseteq V(\mathfrak{a})$.

Now, let $\mathfrak{a}=\left(x_{1}, \ldots, x_{t}\right)$ and let $\varphi: R \rightarrow T$ be the natural epimorphism. As $\mathfrak{a} T=\left(\varphi\left(x_{1}\right), \ldots, \varphi\left(x_{t}\right)\right)$, it follows from Theorem 2 that $\operatorname{Ext}_{T}^{i}(T / \mathfrak{a} T, M)$ is weakly Laskerian $T$-module for all $i$ if and only if the Koszul cohomology modules $H^{i}\left(\varphi\left(x_{1}\right), \ldots, \varphi\left(x_{t}\right) ; M\right)$ are weakly Laskerian $T$-modules for all $i$. But, by above $H^{i}\left(\varphi\left(x_{1}\right), \ldots, \varphi\left(x_{t}\right) ; M\right)$ is a weakly Laskerian $T$-module if and only if $H^{i}\left(\varphi\left(x_{1}\right), \ldots, \varphi\left(x_{t}\right) ; M\right)$ is a weakly Laskerian $R$-module. On the other hand,

$$
H^{i}\left(\varphi\left(x_{1}\right), \ldots, \varphi\left(x_{t}\right) ; M\right) \cong H^{i}\left(x_{1}, \ldots, x_{t} ; M\right) .
$$

Now, the result follows from Theorem 2.

\section{A SUFFICIENT CONDITION FOR $\mathfrak{a}$-WEAKLY COFINITE MODULES}

Theorem 5. Let $f: M \rightarrow N$ be an $R$-homomorphism such that the modules $\operatorname{Ext}_{R}^{i}(R / \mathfrak{a}, \operatorname{Ker} f)$ and $\operatorname{Ext}_{R}^{i}(R / \mathfrak{a}$, Coker $f)$ are both weakly Laskerian for all $i$. Then $\operatorname{KerExt}_{R}^{i}\left(\operatorname{id}_{R / \mathfrak{a}}, f\right)$ and $\operatorname{CokerExt}_{R}^{i}\left(\mathrm{id}_{R / \mathfrak{a}}, f\right)$ are also weakly Laskerian for all $i$.

Proof. Consider the exact sequences

$$
0 \rightarrow \operatorname{Ker} f \rightarrow M \stackrel{g}{\rightarrow} \operatorname{Im} f \rightarrow 0 \text { and } 0 \rightarrow \operatorname{Im} f \stackrel{\iota}{\rightarrow} N \rightarrow \operatorname{Coker} f \rightarrow 0,
$$

where $\iota \circ g=f$. Hence we obtain the following two exact sequences

$$
\cdots \rightarrow \operatorname{Ext}_{R}^{i}(R / \mathfrak{a}, \operatorname{Ker} f) \rightarrow \operatorname{Ext}_{R}^{i}(R / \mathfrak{a}, M) \rightarrow \operatorname{Ext}_{R}^{i}(R / \mathfrak{a}, \operatorname{Im} f) \rightarrow \cdots
$$

and

$$
\cdots \rightarrow \operatorname{Ext}_{R}^{i}(R / \mathfrak{a}, \operatorname{Im} f) \rightarrow \operatorname{Ext}_{R}^{i}(R / \mathfrak{a}, N) \rightarrow \operatorname{Ext}_{R}^{i}(R / \mathfrak{a}, \text { Coker } f) \rightarrow \cdots
$$

Now, since $\operatorname{Ext}_{R}^{i+1}(R / \mathfrak{a}, \operatorname{Ker} f)$ is weakly Laskerian, it follows from the first exact sequence that $\operatorname{CokerExt}_{R}^{i}\left(i d_{R / \mathfrak{a}}, g\right)$ and $\operatorname{KerExt}_{R}^{i+1}\left(i d_{R / \mathfrak{a}}, g\right)$ are both weakly Laskerian for all $i$. Also, as $\operatorname{Ext}_{R}^{i}(R / \mathfrak{a}, \operatorname{Coker} f)$ is weakly Laskerian, the second exact sequence implies that the $R$-modules $\operatorname{CokerExt}_{R}^{i}\left(i d_{R / \mathfrak{a}}, \iota\right)$ and $\operatorname{KerExt}_{R}^{i+1}\left(i d_{R / \mathfrak{a}}, \iota\right)$ are weakly Laskerian for all $i$. Therefore, the assertion follows from the exact sequences

$$
0 \rightarrow \operatorname{KerExt}_{R}^{i}\left(i d_{R / \mathfrak{a}}, g\right) \rightarrow \operatorname{KerExt}_{R}^{i}\left(i d_{R / \mathfrak{a}}, f\right) \rightarrow \operatorname{KerExt}_{R}^{i}\left(i d_{R / \mathfrak{a}}, l\right)
$$


and

$\operatorname{CokerExt}_{R}^{i}\left(i d_{R / \mathfrak{a}}, g\right) \rightarrow \operatorname{CokerExt}_{R}^{i}\left(i d_{R / \mathfrak{a}}, f\right) \rightarrow \operatorname{CokerExt}_{R}^{i}\left(i d_{R / \mathfrak{a}}, \iota\right) \rightarrow 0$.

Corollary 3. Let $M$ be an $R$-module with $\operatorname{Supp} M \subseteq V(\mathfrak{a})$. Suppose that $x \in \mathfrak{a}$ is such that $0: M x$ and $M / x M$ are both $\mathfrak{a}$-weakly cofinite. Then $M$ is also a-weakly cofinite.

Proof. Put $f=x 1_{M}$. Then Ker $f=0:_{M} x$ and Coker $f=M / x M$. Hence in view of Theorem 5, the $R$-module $\operatorname{KerExt}_{R}^{i}\left(1_{R / \mathfrak{a}}, f\right)$ is weakly Laskerian. But since $\operatorname{Ext}_{R}^{i}\left(1_{R / \mathfrak{a}}, f\right)=0$, so $\operatorname{KerExt}_{R}^{i}\left(1_{R / \mathfrak{a}}, f\right)=\operatorname{Ext}_{R}^{i}(R / \mathfrak{a}, M)$. This completes the proof.

Corollary 4. Let $M$ be an R-module. Suppose that $x \in \sqrt{\mathfrak{a}}$ is such that $0: M x$ and $M / x M$ are both weakly Laskerian. Then $\operatorname{Ext}_{R}^{i}\left(R / \mathfrak{a}, \Gamma_{x}(M)\right)$ is also weakly Laskerian for all $i$.

Proof. We have $x^{n} \in \mathfrak{a}$ for some $n \in \mathbb{N}$. Put $f=x^{n} 1_{\Gamma_{x}(M)}$. Then, $\operatorname{Ker} f=$ $0: \Gamma_{x}(M) x^{n}=0: M x^{n}$ and Coker $f=\Gamma_{x}(M) / x^{n} \Gamma_{x}(M)$. Consider the exact sequence

$$
0 \rightarrow \text { Coker } f \rightarrow M / x^{n} M .
$$

As $M / x M$ is weakly Laskerian, it follows from Lemma 2 that $M / x^{n} M$ is weakly Laskerian, and so Coker $f$ is weakly Laskerian. Therefore, in view of [3, Lemma 2.3 (i)] and Theorem 5, $\operatorname{KerExt}_{R}^{i}\left(1_{R / \mathfrak{a}}, f\right)$ is weakly Laskerian. But $x^{n} \in \mathfrak{a}$ implies that $\operatorname{Ext}_{R}^{i}\left(1_{R / \mathfrak{a}}, f\right)=0$, and so $\operatorname{KerExt}_{R}^{i}\left(1_{R / \mathfrak{a}}, f\right)=\operatorname{Ext}_{R}^{i}\left(R / \mathfrak{a}, \Gamma_{x}(M)\right)$. This completes the proof.

Corollary 5. Let $M$ be an $R$-module with $\operatorname{Supp} M \subseteq V(\mathfrak{a})$. Suppose that $x \in \sqrt{\mathfrak{a}}$ is such that $0:_{M} x$ and $M / x M$ are both weakly Laskerian. Then $M$ is a-weakly cofinite.

Proof. The result follows from Corollary 4.

\section{COHOMOLOGICAL DIMENSION AND WEAKLY LASKERIAN MODULES}

Before bringing the next result we recall that, for an $R$-module $M$, the cohomological dimension of $M$ with respect to an ideal $\mathfrak{a}$ of $R$ is defined as

$$
\operatorname{cd}(\mathfrak{a}, M)=\sup \left\{i \in \mathbb{Z} \mid H_{\mathfrak{a}}^{i}(M) \neq 0\right\} .
$$

Proposition 1. Let $\operatorname{cd}(\mathfrak{a})=1$, and let $M$ be a weakly Laskerian $R$-module. Then $\mathrm{H}_{\mathfrak{a}}^{j}(M)$ is $\mathfrak{a}$-weakly cofinite for all $i$. 
Proof. Since $\mathrm{H}_{\mathfrak{a}}^{0}(M)$ is a submodule of $M$, it follows that $\mathrm{H}_{\mathfrak{a}}^{0}(M)$ is a-weakly cofinite. Also, $\operatorname{cd}(\mathfrak{a})=1$ implies that $\mathrm{H}_{\mathfrak{a}}^{i}(M)=0$ for all $i>1$. Therefore, the result follows from [4, Theorem 3.1].

Proposition 2. Let $\mathfrak{b} \supseteq \mathfrak{a}$ be two ideals of $R$ with $\operatorname{cd}(\mathfrak{b})=1$, and let $M$ be an $R$-module with $\Gamma_{\mathfrak{a}}(M)=0$. Then

$$
\mathrm{H}_{\mathfrak{b}}^{j}\left(\mathrm{H}_{\mathfrak{a}}^{i}(M)\right) \cong \begin{cases}\mathrm{H}_{\mathfrak{b}}^{1}(M), & \text { if } \mathrm{j}=0, \mathrm{i}=1 \\ 0, & \text { otherwise. }\end{cases}
$$

Proof. See the proof of [6, Proposition 3.15].

Corollary 6. Let $\mathfrak{b} \supseteq \mathfrak{a}$ be two ideals of $R$ with $\operatorname{cd}(\mathfrak{b})=1$, and let $M$ be a weakly Laskerian R-module. Then $\mathrm{H}_{\mathfrak{b}}^{j}\left(H_{\mathfrak{a}}^{i}(M)\right)$ is $\mathfrak{b}$-weakly cofinite for all $i$ and $j$.

Proof. Since $\operatorname{cd}(\mathfrak{b})=1$, it follows from Proposition 1 that $\mathrm{H}_{\mathfrak{b}}^{j}\left(\Gamma_{\mathfrak{a}}(M)\right)$ is $\mathfrak{b}$-weakly cofinite for all $j$. Now, let $i>0$. As $\mathrm{H}_{\mathfrak{a}}^{i}(M) \cong \mathrm{H}_{\mathfrak{a}}^{i}\left(M / \Gamma_{\mathfrak{a}}(M)\right)$, we may therefore assume that $\Gamma_{\mathfrak{a}}(M)=0$. Thus, the result follows from Propositions 1 and 2 .

Corollary 7. Let $\mathfrak{b} \supseteq \mathfrak{a}$ be two ideals of $R$ with $\operatorname{cd}(\mathfrak{b})=1$, and let $M$ be a weakly Laskerian $R$-module. Then for every finitely generated $R$-module $L$ with $\operatorname{Supp} L \subseteq$ $V(\mathfrak{b})$, the R-modules $\operatorname{Ext}_{\mathrm{R}}^{\mathrm{j}}\left(\mathrm{L}, \mathrm{H}_{\mathfrak{a}}^{\mathrm{i}}(\mathrm{M})\right)$ and $\operatorname{Tor}_{\mathrm{j}}^{\mathrm{R}}\left(\mathrm{L}, \mathrm{H}_{\mathfrak{a}}^{\mathrm{i}}(\mathrm{M})\right)$ are weakly Laskerian for all $i$ and $j$. In particular, the $R$-modules $H_{\mathfrak{a}}^{i}(M) / \mathfrak{b}^{n} H_{\mathfrak{a}}^{i}(M)$ are weakly Laskerian for all $i$ and $n$.

Proof. By Corollary $6, \mathrm{H}_{\mathfrak{b}}^{j}\left(H_{\mathfrak{a}}^{i}(M)\right)$ is $\mathfrak{b}$-weakly cofinite for all $i$ and $j$. Therefore, it follows from Corollary 2 that the $R$-modules $\operatorname{Ext}_{\mathrm{R}}^{\mathrm{j}}\left(\mathrm{R} / \mathfrak{b}, \mathrm{H}_{\mathfrak{a}}^{\mathrm{i}}(\mathrm{M})\right)$ are weakly Laskerian for all $i$ and $j$. Thus, the result follows from Theorem 3 .

\section{ACKNOWLEDGEMENT}

The authors are deeply grateful to the referee for careful reading of the original manuscript and valuable suggestions. The research of the first author was supported in part by a grant from Islamic Azad University, Shabestar Branch.

\section{REFERENCES}

[1] M. P. Brodmann and R. Y. Sharp, Local cohomology. An algebraic introduction with geometric applications, ser. Cambridge Studies in Advanced Mathematics. Cambridge: Cambridge University Press, 1998, vol. 60.

[2] D. Delfino and T. Marley, "Cofinite modules and local cohomology," J. Pure Appl. Algebra, vol. 121, no. 1, pp. 45-52, 1997. 
[3] K. Divaani-Aazar and A. Mafi, "Associated primes of local cohomology modules," Proc. Am. Math. Soc., vol. 133, no. 3, pp. 655-660, 2005.

[4] K. Divaani-Aazar and A. Mafi, "Associated primes of local cohomology modules of weakly Laskerian modules," Commun. Algebra, vol. 34, no. 2, pp. 681-690, 2006.

[5] A. Grothendieck, Local cohomology. A seminar given by A. Grothendieck, Harvard University, Fall 1961. Notes by R. Hartshorne, ser. Lecture Notes in Mathematics. Berlin-Heidelberg-New York: Springer-Verlag, 1967, vol. 41.

[6] L. Melkersson, "Modules cofinite with respect to an ideal," J. Algebra, vol. 285, no. 2, pp. 649-668, 2005.

Authors' addresses

\section{Bahram Vakili}

Department of Mathematics, Islamic Azad University, Shabestar Branch, Shabestar, Iran

E-mail address: bvakil2004@yahoo.com and bvakil@iaushab.ac.ir

\section{Jafar Azami}

Department of Mathematics, University of Mohaghegh Ardabili, Ardabil, Iran

E-mail address: jafar.azami@gmail.com and azami@uma.ac.ir 